\title{
La música de Beethoven en la investigación fisiológica: del electrocardiograma a la imagen por resonancia magnética
}

Beethoven's music inside physiological research: from the electrocardiogram to magnetic resonance imaging

\author{
Miriam Albusac Jorge \\ Universidad de Jaén \\ malbusac@ujaen.es
}

ORCID iD: https://orcid.org/0000-0001-5399-2988

\section{RESUMEN}

La percepción musical produce cambios fisiológicos en los sistemas nerviosos central, autónomo y somático, que pueden verse reflejados en alteraciones del flujo sanguíneo y eléctrico a nivel cerebral, en conjunción con modificaciones de las respuestas cardiovasculares, respiratorias, térmicas, musculares o electrodérmicas, entre otras. La ciencia actual proporciona las herramientas precisas que permiten medir estas variables, ofreciendo un marco único para estudiar la influencia de la música en el ser humano. En este trabajo se analizan las investigaciones que han utilizado la música de L. van Beethoven como estímulo para registrar la actividad biológica humana.

Palabras clave: Beethoven, estímulo, Música, Neurociencia, Psicología. 
LA MÚSICA DE BEETHOVEN EN LA INVESTIGACIÓN FISIOLÓGICA:

DEL ELECTROCARDIOGRAMA A LA IMAGEN POR RESONANCIA MAGNÉTICA

\begin{abstract}
Music perception induces physiological changes in the central, autonomic and somatic nervous systems. These could manifest as alterations in blood and electrical cerebral responses, and cardiovascular, respiratory, thermal, muscular or electrodermal modifications, among others. Current science provides tools to measure these variables, offering a unique framework to study the influence of music on humans. This work analyzes the studies that have used Beethoven's music as stimulus to record human biological activity.
\end{abstract}

Key Words: Beethoven, stimulus, Music, Neuroscience, Psychology.

Albusac Jorge, M. (2020). La música de Beethoven en la investigación fisiológica: del electrocardiograma a la imagen por resonancia magnética. Cuadernos de Investigación Musical, (11, número extraordinario), pp. 216-244.

\title{
1. INTRODUCCIÓN
}

El compositor alemán Ludwig van Beethoven (1770-1827) ha generado un gran corpus de publicaciones y literatura científica a lo largo de la historia de la música. De él se ha estudiado con minuciosidad su vida (Palau, 1945; Rolland, 1915), cada una de las obras de su catálogo (Poggi \& Vallora, 1995), su epistolario (Barrado, 1933), los diversos problemas auditivos que sufrió -tinnitus, hiperacusia y sordera progresiva- (Huxtable, 2001; Perciaccante, Coralli \& Bauman, 2000) y otras de sus variadas patologías y síntomas asociados (Cooper, Nair \& Tremaglio, 2016; Gasenzer \& Neugebaue, 2015; Karmody \& Bachor, 2005), además de cuestiones tan particulares como su capacidad autocrítica (Kozbelt, 2007). En este análisis de su figura han participado incluso otros grandes compositores y teóricos de la música, como Héctor Berlioz, Carl Dahlhaus o Richard Wagner (Berlioz, 1844; Dahlhaus, 1987; Wagner, 1870).

En lo referente a su vida, esta ha sido objeto de múltiples suposiciones y conjeturas llegando a hipotetizar con ideas como la de su envenenamiento (Montes-Santiago, 2013)-, al mismo tiempo que ha inspirado a la producción cinematográfica con películas como Immortal Beloved (Davey \& Rose, 1994), Eroica (Marshall \& Cellan Jones, 2003) o Copying Beethoven (Wilkinson \& Holland, 2006). Más aún: la biografía del músico ha generado hasta guerras dialécticas entre disciplinas, como la surgida entre la Sociología y la Filosofía en torno a su consideración como genio, con posturas de debate enfrentadas que van desde la defensa de su talento innato hasta pensamientos fundamentados en la idea de que, a pesar de su singularidad, las particulares circunstancias sociales y de mecenazgo fueron decisivas en su trayectoria (DeNora, 1995; Kivy, 2001). 


\section{Miriam AlbusaC Jorge}

La música de Beethoven también se ha programado en auditorios de todo el mundo y se ha adaptado para conjuntos instrumentales de diversa índole. Además, sus obras han sido examinadas bajo el prisma de materias heterogéneas, con intervenciones tan variopintas como la de los modelos algorítmicos, estadísticos y de probabilidad (Daikoku, 2018; 2019a; 2019b; Herremans \& Chuan, 2017; Moss, Neuwirth, Harasim \& Rohrmeier, 2019). Asimismo, se han llevado a cabo iniciativas "interactivas" basadas en sus composiciones, como la conferencia-concierto Beethoven and your brain realizada hace una década, donde Daniel Levitin - profesor de Psicología, Neurociencia y Música en la Universidad McGill- y el director de orquesta Edwin Outwater, registraron a través de cuestionarios las diversas respuestas y reacciones del público durante la escucha de fragmentos musicales pertenecientes a la obertura Egmont, Op. 84, y a la Sinfonía n ${ }^{\circ}$ 5, Op. 67, interpretados por la Kitchener-Waterloo Symphony Orchestra, a fin de poner de manifiesto los efectos de la música sobre la audiencia ${ }^{1}$.

A pesar de esta vasta producción sobre el genio, se ha estudiado muy poco el uso que se ha hecho de sus obras como parte de la investigación científica de laboratorio. Por ejemplo, sus composiciones han sido empleadas para realizar mediciones conductuales vinculadas a la interpretación, la escucha y la lectura musical. Así, las seis variaciones para piano en sol mayor sobre el dueto "Nel cor più non mi sento" de la ópera La Molinara de Giovanni Paisello -obra sin número de opus WoO 70-, han sido el punto de partida para estudiar cómo es la ejecución de la música durante la interpretación de notas de adorno, es decir, cuando no existe concreción en la duración ${ }^{2}$, y cómo esta puede afectar al tempo musical (Timmers, Ashley, Desain, Honing \& Windsor, 2002). Del mismo modo, el tercer movimiento de la Sonata Op. 31, $n^{\circ}$, se ha utilizado con el objetivo de comparar los patrones temporales de múltiples interpretaciones de una misma obra -medidos a través de oscilogramas- (Repp, 1990). Sin embargo, no sólo esta sonata para piano de Beethoven ha tenido relevancia en la investigación conductual, sino que varias de ellas -concretamente la $\mathrm{n}^{\circ}$ 4, Op. 7; la no 11, Op. 22; la n ${ }^{\circ}$ 14, Op. 27, $\mathrm{n}^{\circ}$ 2, conocida como "Claro de luna"; la n ${ }^{\circ}$ 21, Op. 53, denominada "Waldstein"; la no 28, Op. 101; la n 17 , Op. 31, n 2, designada "La tempestad"; y la no 32, Op. 111- han sido fundamentales para analizar cuestiones tonales asociadas la percepción musical, factores relacionados con el pulso o las bases psicofísicas de determinados elementos armónicos (Chan, Dong \& Li, 2019; Clynes, 1995; Eitan \& Timmers, 2010; Lalitte, Bigand, Kantor-Martynuska \& Delbé, 2009; Smith \& Cuddy, 2003; Tillmann, Lalitte, Albouy, Caclin \& Bigand, 2016). Igualmente, las bagatelas -Op. 33, Op. 119 y Op. 126- y, de nuevo, una sonata -la Op. 2, n 1-, han servido como estímulo visual en un estudio que examinaba los movimientos oculares de músicos mientras leían partituras, en busca de singularidades (Servant \& Baccino, 1999).

En otros trabajos científicos, lejos de las medidas conductuales vinculadas con el hecho musical, la obra beethoveniana se ha utilizado también en tareas de índole cognitivo. Por

\footnotetext{
1 La información sobre esta experiencia y su programación puede consultarse en http://www.beethovenandyourbrain.com

${ }^{2}$ Las notas de adorno no poseen una duración prefijada, sino que toman su valor de las notas reales a la que acompañan.
} 
ejemplo, la ya citada obertura Egmont-junto con música del compositor W. A. Mozart-, ha acompañado la realización tanto de pruebas psicológicas estandarizadas como de tareas lógico-matemáticas, no hallando mejoras en el rendimiento de las mismas bajo ninguna de las dos condiciones ${ }^{3}$ musicales. De hecho, el tiempo dedicado a estas tareas fue significativamente mayor cuando se solapaban con la escucha de la pieza de Beethoven frente a las demás condiciones del experimento: la obra de Mozart o el silencio ambiental-, al tiempo que se cometían más cantidad de errores. En concreto, los autores atribuyeron este suceso a la complejidad de la música de Beethoven (Mitas, Lipowicz, Wijata \& Bieńkowska, 2016). Además, estudios publicados bajo la vorágine del conocido como "Efecto Mozart"4 han indicado resultados similares para actividades cognitivas que involucran la memoria de trabajo, concluyendo que, independientemente de la autoría de la música que se escucha antes de realizar la tarea -Mozart o Beethoven-, no existen diferencias estadísticamente significativas en la ejecución posterior - en este caso particular, la música de Beethoven era el tercer movimiento del Concierto para violín en re mayor, Op. 61- (Giannouli, Tsolaki \& Kargopoulos, 2010). Sin embargo, este resultado ha sido contradicho por otras investigaciones que han encontrado mejoras en tareas cognitivas de razonamiento espacial ante la exposición a la música mozartiana, pero no así cuando la composición era de Beethoven o había silencio (Suda, Morimoto, Obata, Koizumi \& Maki, 2008).

Dentro de la esfera cognitiva, se ha indagado también sobre el efecto de la música durante el aprendizaje en el entorno académico. En este contexto, la escucha simultánea de una conferencia junto con música como la "Oda a la Alegría" de la Sinfonía n 9, Op. 125, entre otras composiciones, mejoró las respuestas que se ofrecían en un cuestionario realizado con posterioridad, basado en los contenidos de la citada ponencia (Dosseville, Laborde \& Scelles, 2012). Una idea similar subyace en el método de "reactivación de la memoria dirigida", que consiste en asociar una señal externa -en este caso musical- con información recientemente aprendida. Más tarde, ese estímulo estará presente mientras la persona duerme, con lo que se pretende estabilizar y afianzar en la memoria la nueva información adquirida. Así, aplicando este método, se ha empleado el primer movimiento de "Claro de luna" -junto con obras de Vivaldi y Chopin- para promover la integración de conceptos educativos de nivel universitario, indicando que la música puede mejorar el rendimiento en

\footnotetext{
${ }^{3}$ Cuando se realiza un estudio científico, normalmente se maneja una variable - denominada independientecon el objetivo de analizar los efectos que esta provoca sobre una segunda variable -llamada dependiente-. En las investigaciones que se recogen en este texto, la música es la variable independiente, mientras que la variable dependiente sería aquella que se mide y cuantifica - por ejemplo, el rendimiento cognitivo o las respuestas fisiológicas-. La variable independiente suele tener diferentes niveles o condiciones del estudio, que representan tratamientos distintos de la misma. Por ejemplo, en un estudio médico para conocer la eficacia de un fármaco, tomar las pastillas " $\mathrm{X}$ ", "Y" o las píldoras placebo, podrían ser tres condiciones de la variable independiente “medicación”. En el contexto de este artículo, escuchar música de Beethoven, composiciones de otros autores, sonidos de la naturaleza o tener un entorno en el que los estímulos auditivos están ausentes, serían ejemplos hipotéticos de cuatro condiciones.

${ }^{4}$ El "Efecto Mozart" aparece a raíz de un artículo publicado en la revista Nature en el año 1993, que indicaba que la escucha previa de la Sonata para dos pianos en re mayor K. 448 de Mozart mejoraba a corto plazo la capacidad de razonamiento abstracto y las habilidades espacio-temporales en una actividad posterior (Rauscher, Shaw \& Ky, 1993).
} 


\section{Miriam AlbusaC Jorge}

una prueba de conocimientos realizada al día siguiente de la adquisición de la información (Gao, Fillmore \& Scullin, 2020). Además, otras investigaciones también han hecho uso de esta sonata y de la $\mathrm{n}^{\circ} 6$, Op. 10, $\mathrm{n}^{\circ}$ 2, para examinar las relaciones entre cambios emocionales y cognición (Hausmann, Hodgetts \& Eerola, 2016).

En definitiva, la obra de Beethoven ha sido estímulo para diversas modalidades sensoriales, como la auditiva o la visual. Sin embargo, su utilización no sólo se ha ceñido a la investigación en humanos, sino que también ha estado presente en estudios con animales; por ejemplo, la pieza "Para Elisa", se ha empleado al objeto de analizar el rendimiento de ratones dentro de un laberinto - medido a través del registro tanto del tiempo de respuesta como de los errores cometidos- mientras escuchaban la composición (Aoun, Jones, Shaw \& Bodner, 2005).

La idea de que la música tiene un influjo sobre los seres vivos y, en especial, sobre el ser humano, ha sido una temática recurrente desde los albores de la humanidad y, ya en la antigüedad griega, fue plasmada en la teoría del ethos. No en vano, junto con el desarrollo de las múltiples técnicas de registro de las funciones biológicas -y al margen de las medidas cognitivo-conductuales-, también ha aparecido un gran interés por cuantificar los efectos de la música. Así, desde el ámbito de la Psicología, la Neurociencia y otras disciplinas, se han examinado los cambios orgánicos provocados por la percepción de diferentes tipologías de estímulos musicales con fines diversos. En esta línea, el presente texto analiza las investigaciones que vinculan la escucha de las obras de Beethoven con distintas respuestas fisiológicas ${ }^{5}$ en humanos, detallando qué piezas son, para qué fin se han empleado y los resultados más relevantes en cuanto a ellas.

\section{LA PERCEPCIÓN MUSICAL Y SU INFLUENCIA}

El cuerpo humano responde de múltiples formas al recibir un estímulo auditivo musical. Cuando un sonido llega al tímpano, se ponen en marcha una serie de mecanismos de procesamiento periférico y central que, a través de sucesos mecánicos, químicos y neurales en diversas estructuras como la cóclea, el tronco cerebral, los núcleos del cerebro medio o la corteza auditiva, dan lugar a la percepción (Peretz \& Zatorre, 2005, p. 90). Según el diccionario de la Real Academia Española, la percepción se define como la "sensación interior que resulta de una impresión material hecha en nuestros sentidos" (2019) -segunda acepción-. Existen otras definiciones para este término como, por ejemplo, la de Enghels \& Roegiest, quienes indican que se trata de "un tipo particular de proceso cognitivo por el que un individuo [...] experimenta un estímulo exterior y obtiene por ese estímulo información sobre el mundo" (2004, p. 47).

\footnotetext{
${ }^{5}$ Respuestas fisiológicas entendidas en el sentido amplio del término, pudiendo definirse como reacciones del organismo ante un estímulo determinado, que abarcan desde variables de fácil obtención -como la frecuencia cardíaca-, hasta cambios fisiológicos cerebrales registrados con técnicas de neuroimagen.
} 
En el contexto de este artículo es fundamental conocer los procesos que desencadena la percepción musical, así como los sustratos cerebrales vinculados a ella, para una mejor comprensión de los resultados ofrecidos por las investigaciones que han utilizado la música de Beethoven en relación a la fisiología, que se describirán en un epígrafe posterior.

La percepción musical involucra funciones cerebrales complejas del sistema nervioso (SN) central (Koelsch \& Siebelb, 2005, p. 578) e influye en los sistemas nerviosos autónomo y somático ${ }^{6}$. Para examinar las diferentes respuestas biológicas asociadas a la música, se emplean técnicas de registro fisiológico que permiten medir de forma no invasiva aquellas actividades del organismo que dependen del SN, ya sea de forma directa o indirecta (Redolar Ripoll, 2010, p. 113). En las clasificaciones tradicionales de estas técnicas, suele tenerse en cuenta el tipo de sistema que media en cada una de las respuestas: SN central, autónomo o somático. En el primer caso, la fisiología cerebral o neurofisiología puede registrarse mediante técnicas que reflejan la actividad eléctrica -el electroencefalograma o la magnetoencefalografía se encuadran esta categoría- y hemodinámica -tales como la tomografía por emisión de positrones o la resonancia magnética funcional-, entre otras. En las mediciones del SN autónomo suele emplearse, aunque no en exclusiva, el electrocardiograma para la frecuencia cardíaca o el esfigmomanómetro para la tensión arterial, además de diversos dispositivos para medir la actividad electrodérmica de la piel también denominada conductancia o respuesta galvánica- y otras múltiples herramientas. Por último, en el caso del SN somático, las técnicas de registro más utilizadas son el electrooculograma y el electromiograma -para examinar la actividad de los músculos circundantes a los ojos o de los músculos esqueléticos, respectivamente- (Redolar Ripoll, 2010, p. 114).

En lo referente a la percepción musical, cabe poner de relieve cómo las técnicas de registro de la actividad cerebral han mostrado que existe un trabajo complementario de diferentes regiones y mecanismos cerebrales -donde a veces participa predominantemente un hemisferio, mientras que otras veces se requiere de intervenciones bilaterales- que, de forma conjunta, se encargan del procesamiento de la información musical (Peretz \& Zatorre, 2005). Así, los estímulos musicales de entrada son segregados y analizados en sus dimensiones individuales y, aunque la comprensión del mensaje musical es interconectada y global, diversas áreas cerebrales se encargan de descodificar las diferentes cualidades de la música. Por tanto, no existe un centro cerebral único donde procesar la música, sino que median redes disgregadas por todo el cerebro:

\footnotetext{
${ }^{6}$ El SN de un organismo está compuesto por todas sus neuronas, junto con las células que le sirven de soporte. Este puede dividirse en SN Central y SN Periférico, estando el primero integrado por el encéfalo y la médula espinal y el segundo por los nervios y las estructuras denominadas ganglios nerviosos (Kiernan, 2013, pp. 3-4). Formando parte del SN periférico se encuentra el SN autónomo o vegetativo, que se ocupa principalmente de controlar las funciones involuntarias como pueden ser la sudoración o las actividades cardiovascular, pupilar o gastrointestinal. En el SN periférico se encuentra también incluido el SN somático, encargado en este caso de regular las funciones voluntarias y gestionar la información sensorial que llega al espécimen.
} 


\section{Miriam AlbuSAC JORGE}

Oír, interpretar o componer música [...] exige la participación de casi todo el subsistema neuronal $[. .$.$] . La música se distribuye por todo el cerebro, en contra de la antigua idea$ simplista de que el arte y la música se procesan en el hemisferio derecho, mientras que el lenguaje y las matemáticas se procesan en el izquierdo (Levitin, 2008, p. 17).

Las investigaciones realizadas en personas con daños selectivos han indicado la existencia de una arquitectura modular para el procesamiento de la música, que abarca diferentes componentes o módulos, con vías de circulación de la información entre ellos (Koelsch, 2011; Peretz \& Coltheart, 2003). Estos módulos tienen sus propios procedimientos, estando asociados con sustratos neurales especializados y separados (Peretz \& Coltheart, 2003, p. 688; Warren, 2008). De esta forma, como cada módulo tendría una función concreta, un daño en un área específica del cerebro no afectaría a las habilidades musicales de forma global, sino que dañaría individualmente el procesamiento de una dimensión concreta (Carmona \& Daza, 2008, p. 89); por ejemplo, podría verse dañada la percepción rítmica, pero no así la codificación tonal. En este sentido, según Fordor -autor que ha tratado extensamente los principios de la modularidad-, los sistemas modulares son "específicos y autónomos, o sea, que siempre están referidos a representaciones particulares y concretas, las cuales pueden tratarse con total independencia de otros sistemas funcionales de carácter no modular” (Montilla López, 1999, p. 112). En otras palabras:

Una estructura modular es, en síntesis, aquella que es capaz de detectar errores y corregirlos sin necesidad de recurrir para ello a ninguna otra estructura de tipo no modular. Las partes que constituyen los sistemas modulares son los módulos, celdas específicas para un dominio concreto de la cognición, pero encapsuladas en el plano de la información, lo que es equivalente a decir que los módulos únicamente realizan los tratamientos que les son propios, lo que tampoco se corresponde necesariamente con que unos módulos sean independientes de otros (Montilla López, 1999, pp. 112-113).

En general, la literatura apunta hacia el procesamiento independiente de la organización tonal y temporal en la música (Peretz \& Zatorre, 2005, p. 91). Así, para las cuestiones tonales existen módulos de codificación tonal, distinción de intervalos y examen del contorno melódico, al tiempo que en el dominio temporal se integran los módulos de análisis rítmico y métrico (Peretz \& Coltheart, 2003, p. 690). Ciertos componentes de esta arquitectura modular se encargan únicamente de procesar estímulos musicales, mientras que otros tendrían a la vez distintas funcionalidades. De este modo podría explicarse cómo la música y el lenguaje no dependen cerebralmente de circuitos idénticos, aunque sí podrían existir módulos compartidos por ambos y superposición neuronal para determinadas funciones (Peretz, Vuvan, Lagrois \& Armony, 2015). A continuación, se describe de forma más pormenorizada la relación de los elementos musicales con los sustratos neurales anatómicos y funcionales. 
La corteza auditiva primaria está situada en el lóbulo temporal -dentro de la fisura de Silvio-, concretamente en la parte medial de la circunvolución de Heschl (Warren, 2008, p. 34). En lo referente a los tonos, hay que señalar que el cerebro no los percibe como entes aislados, salvo en el caso de las personas con la capacidad del oído absoluto. Por el contrario, es la secuenciación la que permite diferenciar entre un tono y el inmediato posterior, distinguiendo la distancia relativa entre los mismos (Barquero Jiménez \& Payno Vargas, 2001, p. 456). La corteza temporal derecha tiene un papel muy importante para el procesamiento tonal: de hecho, las personas con daño focal temporal en el hemisferio derecho muestran más déficits en la esfera tonal que los sujetos con lesión temporal izquierda (Peretz \& Zatorre, 2005, p. 91). En términos de localización neuronal, es la porción lateral de la circunvolución de Heschl la que se ocupa principalmente del análisis tonal (Patterson, Uppenkamp, Johnsrude \& Griffiths, 2002; Warren, 2008, p. 34). En consecuencia, después de un daño en esta circunvolución -en el hemisferio derecho-, se han observado déficits en ciertos aspectos del procesamiento tonal (Peretz \& Zatorre, 2005, p. 91; Zatorre, 1988). Además:

\begin{abstract}
Alrededor del giro de Heschl existe una red de áreas corticales superiores que están distribuidas a lo largo de los lóbulos temporal, parietal y frontal. Esta red incluye "áreas de asociación" auditiva, que se encargan de procesar ciertas propiedades de los sonidos complejos. Por detrás del giro de Heschl se encuentra el plano temporal [...], un área de asociación auditiva que está implicada en el análisis de diferentes atributos de las fuentes de sonido complejas, incluida su ubicación espacial [...] o el timbre de una voz o instrumento musical, e información sobre patrones tonales ${ }^{7}$ (Warren, 2008, p. 34).
\end{abstract}

Por otro lado, hay que señalar que la circunvolución temporal superior de ambos lóbulos se ocupa primordialmente del análisis melódico, mientras que circuitos en las regiones parietal y frontal median en la memoria de trabajo musical (Warren, 2008, p. 34). En definitiva, el área auditiva primaria derecha parece ser esencial para la representación de la información tonal, mientras que el procesamiento de melodías requiere de intervención bilateral -con predominio del hemisferio derecho-, participación de áreas corticales de orden superior y conexiones con la corteza frontal (Zatorre, 2001, p. 193).

En lo que concierne a la percepción del timbre hay que destacar cómo el lóbulo temporal superior derecho tiene también un papel muy predominante, aunque su equivalente izquierdo participa igualmente en el procesamiento: principalmente se ha encontrado implicación de regiones temporales anteriores y posteriores (Samson, 2003, p. 144). Debido a ello, los problemas para el reconocimiento tímbrico han sido asociados de igual modo con

\footnotetext{
${ }^{7}$ Texto original: "Surrounding HG is network of higher cortical areas in the temporal, parietal and frontal lobes. This network includes auditory 'association areas' that process certain properties of complex sounds. Posterior to HG lies the planum temporale [...], an auditory association area that has been implicated in the analysis of different attributes of complex sound sources, including their spatial location [...], or the timbre of a voice or musical instrument, and information about pitch patterns they carry" (Traducción propia).
} 


\section{Miriam AlbusaC Jorge}

déficits en el ámbito tonal, aunque pueden ocurrir dificultades selectivas para el procesamiento del timbre como consecuencia de lesiones en la circunvolución temporal (Warren, 2008, p. 35).

Respecto a la organización temporal de la música puede afirmarse, en general, que mientras que la corteza auditiva del hemisferio derecho mantiene la supremacía para los rasgos tonales, la izquierda se ocupa especialmente de la dimensión temporal (Abecasis, Brochard, Del Río, Dufour \& Ortiz, 2009, p. 77). La percepción del ritmo es básica para la experiencia relacionada con la estructura temporal de la música y ha sido extensamente estudiada por la Psicología (Snyder, Large \& Penhune, 2009, p. 13). En concreto, el ritmo de la música se ha vinculado a las cortezas motora y premotora, al cerebelo y a los ganglios de la base, estructuras que también están implicadas en el movimiento corporal (Grahn, 2012, p. 593). A este respecto, ya en la antigüedad griega, Platón exponía la estrecha imbricación entre el ritmo y el movimiento, definiendo este como el orden de los movimientos (Martín Moreno, 2003, p. 324). Además, parece que la percepción del ritmo depende de las interacciones entre el sistema auditivo y el motor: el acoplamiento entre ambas regiones es tan sumamente crucial que, pacientes con lesiones en la corteza auditiva, han mostrado problemas rítmicos en tareas de reproducción y sincronización motora, a pesar de tener intacto el sistema motor (Chen, Penhune \& Zatorre, 2009, p. 16). En relación a las ideas expuestas en este párrafo, algunas propuestas han indicado también un importante rol de los ganglios de la base en la generación interna de la sensación de pulso (Grahn, 2009; 2012, p. 593). Por último, en cuanto a las características emocionales asociadas a la música, hay que destacar que su procesamiento implica principalmente el sistema límbico.

En definitiva, todas las dimensiones de la música se procesan en la corteza cerebral y en algunas regiones subcorticales -como ocurre con la emoción-, interviniendo los dos hemisferios y siguiendo un patrón modular, dentro de una red global interconectada. En otras palabras y recapitulando las ideas de este epígrafe, cuando tiene lugar la percepción musical se ponen en marcha diversas y distantes regiones cerebrales, que suponen cambios en la actividad eléctrica y hemodinámica del sistema nervioso central. Al mismo tiempo, la percepción produce otras reacciones corporales y emocionales dependientes de los sistemas nerviosos autónomo y somático. Estas últimas se traducen en modulaciones de las frecuencias cardíaca y respiratoria, la presión arterial, la oxigenación de la sangre, la temperatura, la respuesta galvánica de la piel, el reflejo pupilar y múltiples actividades musculares como puede ser la cigomática (ejemplos en: Bullack, Büdenbender, Roden \& Kreutz, 2018; Grewe, Nagel, Kopiez \& Altenmüller, 2007; Hodges, 2010; Iwanaga \& Moroki, 1999; Khalfa, Peretz, Blondin \& Robert, 2002; Krumhansl, 1997; Lundqvist, Carlsson, Hilmersson \& Juslin, 2009; Merrill, Omigie \& Wald-Fuhrmann, 2020; Nyklíček, Thayer \& Van Doornen, 1997; Orini et al., 2010). 


\section{LAS OBRAS DE BEETHOVEN COMO ESTÍMULO AUDITIVO EN LA INVESTIGACIÓN FISIOLÓGICA}

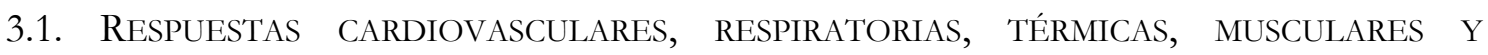 ELECTRODÉRMICAS}

En el histórico de investigaciones que han estudiado las respuestas fisiológicas vinculadas a la música, las primeras hipótesis cuestionaron si la modificación de distintos parámetros musicales, tales como el tempo o la intensidad, supondría también de forma análoga una variación en las respuestas cardiovasculares y respiratorias. De igual manera, ha sido recurrente analizar cómo fragmentos musicales con gran número de disonancias o, por el contrario, mayoritariamente consonantes, actuarían en este sentido. Además, también se ha estudiado si las piezas musicales categorizadas por los participantes como "preferidas", o definidas subjetivamente como "tristes"/“alegres" o "excitantes"/“tranquilizantes", pueden influir sobre estas medidas biológicas y cómo lo hacen (Bullack et al., 2018; Iwanaga \& Moroki, 1999).

Desde los inicios del siglo XX e, incluso antes, se viene informando de variaciones en el electrocardiograma, la presión arterial y la respiración debidos a la música (Ellis \& Brighouse, 1952; Hyde, 1924; Hyde \& Scalapino, 1918). Los estudios en este campo han utilizado piezas de diversos géneros y estilos musicales, entre ellas, obras compuestas por Beethoven. Así, el "Adagio molto e cantabile" de la Sinfonía n” 9, Op. 125, interpretado con una velocidad de 70 pulsos de negra por minuto, se ha empleado para comprobar la influencia de los cambios de tempo en las variables respiratoria y cardiovascular, junto con otra serie de obras de heterogéneas velocidades y estilos. Los resultados mostraron cómo estas variables se modificaban dependiendo de la velocidad de la música más que del estilo en sí: los tempos más rápidos provocaron aumentos significativos de la frecuencia respiratoria, de la ventilación, de la frecuencia cardíaca y de la presión arterial -sistólica y diastólica-. Además, estos incrementos eran independientes de las preferencias musicales de los sujetos y no fueron condicionados por las mismas. Concretamente, en el caso del "Adagio molto e cantabile" de Beethoven, se produjeron respuestas cardiorrespiratorias reducidas (Bernardi, Porta \& Sleight, 2006).

La Sinfonía $n^{\circ} 9$-tercer movimiento completo- también se ha destinado, junto a obras de otros compositores, a analizar cómo responden las personas ante los cambios de intensidad de la música. Específicamente, en un estudio sobre esta cualidad sonora, los fragmentos seleccionados fueron estos: el aria "Nessun dorma" de la ópera Turandot de G. Puccini -secciones con crescendos vocales progresivos-; el citado "Adagio" de la Sinfonía n 9 partes que contienen también aumentos progresivos de intensidad, pero orquestales en este caso-; la Cantata BWV 169, "Gott soll allein mein Herz haben”, de J. S. Bach -como ejemplo de énfasis dinámico uniforme-; y frases tomadas de las arias de G. Verdi “Va pensiero" y "Libiam nei lieti calci". Los datos revelaron que prácticamente todos los crescendos, tanto vocales como 


\section{Miriam Albusac Jorge}

orquestales -incluidos, por tanto, los del mencionado "Adagio" de la Sinfonía n" 9-, produjeron correlaciones positivas entre la intensidad de la música y las variables cardiovasculares y respiratorias -implicando vasoconstricción de la piel y aumentos tanto de la presión arterial como de la frecuencia cardíaca-, siendo estas proporcionales a la magnitud del crescendo. Por el contrario, la intensidad más uniforme se tradujo en vasodilatación cutánea y disminución de la presión arterial. En consecuencia, las respuestas fisiológicas parecían ajustarse al patrón de intensidad musical que imperaba en cada momento, sincronizándose con él (Bernardi et al., 2009; Trappe, 2010; 2012a; 2012b).

Los dos estudios descritos hasta el momento en este epígrafe se realizaron en adultos. No obstante, las modificaciones de estas variables biológicas en respuesta a la música ocurren también en otros grupos de población, como pueden ser los bebés. Así, en una investigación con recién nacidos sanos, se recurrió a la Sonata "Claro de luna" que, junto con una composición de Mozart se aplicó como estímulo musical, acompañando la realización de pruebas médicas dolorosas -por ejemplo, inyecciones intramusculares-. Además, se utilizó un tercer estímulo, que era una grabación de latidos del corazón a 70 pulsaciones por minuto. Las conclusiones revelaron que los bebés, cuando escuchaban la música o los sonidos del corazón, manifestaban reducción de la frecuencia cardíaca, al mismo tiempo que un aumento en la saturación de oxígeno. Sin embargo, esto no sucedió en la condición de silencio del grupo control, que no tuvo estimulación auditiva (Rossi et al., 2018). Específicamente, la frecuencia cardíaca media descendió de 112,35 latidos por minuto -lpm-a 110,7 lpm en el grupo que escuchó la música de Mozart; de 119,8 a 110,8 lpm en el grupo que estaba estimulado por la sonata de Beethoven; y de 111,8 a 105,6 lpm en el caso de aquellos que oían la grabación de los latidos del corazón. Esto suponía un cambio promedio de 1,64lpm cuando se escuchaba a Mozart; 6,2 lpm con la grabación de los latidos; y hasta 9 lpm en el grupo estimulado con "Claro de luna". En contraposición, la frecuencia cardíaca aumentó de 124,2 a 128,75 lpm en el grupo de control. Por su parte, la saturación media de oxígeno varió un $0,95 \%$ y un $0,7 \%$ en los grupos que escucharon a Mozart y los latidos, respectivamente, mientras que en el grupo al que se le asignó la música de Beethoven la variación fue del 1,4\%. En definitiva, con este estudio se demostró que la exposición tanto a música como a grabaciones de los latidos del corazón puede modificar los parámetros fisiológicos a corto plazo en recién nacidos, con las implicaciones que de ello pueden derivarse en lo referente a la reducción del estrés en situaciones dolorosas (Rossi et al., 2018).

En relación al estrés, hay que señalar que tanto este como la ansiedad pueden verse reflejados en diversas variables fisiológicas. Por ello, algunos estudios han tratado de evaluar el efecto de la música sobre estas problemáticas empleando composiciones de Beethoven (Lai et al., 2008; Son, So \& Kim, 2019). Concretamente, en una investigación sobre ello, la Sonata "Claro de luna" fue de nuevo seleccionada, junto con el Romance para violín $n^{\circ}$ 2, Op. 50, de Beethoven, y otras composiciones de Mozart y Haydn, con el objetivo de influir sobre la ansiedad producida ante un examen en estudiantes de enfermería. Los resultados indicaron eficacia de la música, originando reducción en la frecuencia del pulso y la presión arterial, a la vez que un aumento de la temperatura de los dedos (Lai et al., 2008). 
De igual forma y, aunque el rol de la música para la disciplina médica aún no está claro, en el ámbito clínico se han realizado otros trabajos que indican posibles mejoras en el sistema inmunológico tras la escucha tanto de la Sonata "Claro de luna" como del Concierto para piano $n^{\circ} 4$ en sol mayor, Op. 58 (Trappe, 2012b, p. 30). Además, también se ha observado cierta ayuda en los estados depresivos empleando como estímulo auditivo el ya mencionado Concierto $n^{\circ}$ 4, junto con la Sinfonía n ${ }^{\circ}$ 5, Op. 67 (Chan, Chan, Mok \& Kwan Tse, 2009; Trappe, 2012b, p. 30). En particular, un estudio reveló que la escucha de la Sinfonía $n^{\circ} 5$ de Beethoven durante un mes en personas mayores -junto con jazz y música clásica tanto china como asiáticasupuso una disminución en las puntuaciones psicológicas de depresión, además de en la presión arterial y las frecuencias cardíaca y respiratoria (Chan et al., 2009).

Para concluir este apartado y, aunque se trata de un estudio que no utiliza un estímulo en la modalidad auditiva, resulta interesante señalar las conclusiones que aportan Yoshie y sus colaboradores en lo referente a la ansiedad que se produce durante la interpretación musical en músicos expertos. En este caso, se hicieron mediciones tanto del estado subjetivo de ansiedad como de la frecuencia cardíaca, la frecuencia de sudoración y la actividad electromiográfica de los músculos de las extremidades superiores, durante la ejecución musical al piano de sonatas de Beethoven -entre ellas, la Sonata $n^{\circ} 23, O p$. 57-, Prokofiev y Rachmaninov, que eran elegidas por cada sujeto. La interpretación se producía en dos condiciones: una estresante -situación en la que competían delante de un jurado experto y audiencia- y una no estresante -ensayo-. Entonces, el electrocardiograma, electromiograma y el medidor de tasa de sudoración revelaron que, frente a los ensayos, en la condición estresante había una mayor frecuencia cardíaca, resistencia de la piel y magnitud de electromiografía, junto con una elevada ansiedad subjetiva y gran número de errores en la interpretación (Yoshie, Kudo, Murakoshi \& Ohtsuki, 2009).

\subsection{EL MOVIMIENTO CEREBRAL}

\subsubsection{ELECTROENCEFALOGRAMA}

La percepción musical, como se ha expuesto en un epígrafe anterior, también moviliza los recursos cerebrales. De hecho, los procesos neuronales subyacentes a la escucha musical han sido investigados a través de la electroencefalografía. Entre los estímulos empleados, la música de Beethoven ha ocupado un espacio en los estudios que examinan la actividad eléctrica del cerebro con esta herramienta (Hamada, Zaidan \& Zaidan, 2018). Por ejemplo, tanto el primer movimiento de "Claro de luna" como el tercero de la Sinfonía n" 6, Op. 68, también conocida como "Pastoral", han formado parte de un conjunto de 40 extractos instrumentales - de un minuto de duración- pertenecientes a diversos géneros y compositores, con el propósito de investigar las emociones. Los resultados probaron excitación en áreas del cerebro vinculadas con las emociones, "principalmente en la proximidad de las regiones tálamo-límbica y orbitofrontal, así como en las áreas frontal, 
fronto-parietal, parietal, parieto-occipital, temporo-occipital y occipital"» (Rogenmoser, Zollinger, Elmer \& Jäncke, 2016, p. 1428). Además, como las emociones eran variadas en cuanto a valencia ${ }^{9}$ y grado de excitación, esta última se asoció con una supresión del poder de la frecuencia alfa, mientras que la valencia lo hizo con un aumento de la frecuencia theta -en el caso de extractos "felices" o que pueden llegar a inducir esa emoción, en comparación con los “tristes"- (Rogenmoser et al., 2016, p. 1428).

Los estudios desarrollados en el marco de la disciplina conocida bajo el nombre de Neurociencia de la Música, además de estudiar la percepción musical o las relaciones entre la música y la emoción, también han hecho especial énfasis en las modificaciones cerebrales estructurales y funcionales-, asociadas al entrenamiento musical: en otras palabras, a la plasticidad cerebral vinculada con la práctica interpretativa. Así, se ha corroborado cómo los músicos profesionales muestran una actividad neuroeléctrica diferencial cuando perciben la música. En relación a ello, se planteó un estudio en el que músicos aficionados y profesionales escuchaban el primer movimiento completo de la Sinfonía $n^{\circ} 5$ de Beethoven, Op. 67, con el objetivo de examinar diferencias emocionales en el nivel de excitación autoinformado. Los resultados indicaron que los músicos mostraban patrones cerebrales diferentes en comparación con los aficionados, por ejemplo, en la actividad theta frontalmedial, durante la percepción musical. Además, durante los picos máximos de excitación, los músicos manifestaban también un aumento en la actividad alfa posterior, delta central y beta (Mikutta, Maissen, Altorfer, Strik \& Koenig, 2014). Otro estudio de este grupo de autores, basado también en el primer movimiento completo de la Sinfonía $n^{\circ} 5$, indicaba que esta música era un estímulo óptimo para modular el nivel de excitación, siendo capaz de producir "cambios consistentes en los estados funcionales del cerebro asociados con la excitación y las emociones" $"$, independientemente de las preferencias individuales de cada sujeto y de sus diferencias (Mikutta, Altorfer, Strik \& Koenig, 2012, pp. 428-429).

Empleando electroencefalografía también se han analizado posibles diferencias tanto en músicos con formación clásica como en músicos vinculados con el rock, cuando estos escuchaban su género preferido -música clásica versus rock- durante la realización de una tarea visual (Caldwell \& Riby, 2007). La obra elegida para ilustrar la condición de música clásica fue la Sinfonía $n^{\circ}$ 2, Op. 36, de Beethoven. Los datos mostraron amplitudes en determinados componentes eléctricos cuando los participantes escuchaban música vinculada con la formación que habían recibido y para la que se habían especializado. En concreto, los componentes alterados estaban asociados con la memoria de trabajo consciente, es decir, con el procesamiento cognitivo activo, por lo que una reducción en los mismos podría indicar que se estaban empleando menos recursos cognitivos (Hurless et al., 2013). Además, se

\footnotetext{
8 Texto original: "namely in the proximity of thalamic-limbic and orbitofrontal regions as well as at frontal, fronto-parietal, parietal, parieto-occipital, temporo-occipital and occipital areas” (Traducción propia).

${ }^{9} \mathrm{La}$ valencia emocional se emplea para clasificar las emociones y hace referencia a sus características. En este sentido, emociones como el miedo, la tristeza o la ira, tendrían una valencia negativa, mientras que, en contraposición, la alegría asumiría una valencia positiva.

10 Texto original: "consistent changes in brain functional states associated with arousal and emotions" (Traducción propia).
} 
observaron también efectos beneficiosos de la exposición a la música beethoveniana, para ambos grupos, en la etapa pre-atencional del procesamiento de la información (Caldwell \& Riby, 2007, p. 992).

En lo que respecta a personas sin formación musical, también se ha investigado el efecto de la música de Beethoven sobre la actividad cerebral, en particular en adultos y ancianos sanos y en ancianos con deterioro cognitivo leve. En este caso, al escuchar "Para Elisa", no se notificaron alteraciones en la actividad neuroeléctrica en los adultos y los ancianos, aunque sí había modificaciones en la banda alfa cuando la obra era de Mozart resultados preliminares-. Este patrón de actividad podría estar indicando la activación de circuitos neuronales relacionados con procesos cognitivos como la memoria, la atención o la resolución de problemas (Verrusio et al., 2015).

Por último, otro ejemplo del uso de las obras de Beethoven en conjunción con electroencefalograma aparece en un ensayo clínico en epilepsia infantil donde, también a través de la obra "Para Elisa" -que se escuchaba en bucle durante 18 minutos- y de una composición de Mozart, se investigó el efecto de la música sobre la frecuencia de descargas epileptiformes. No obstante, el estudio, aunque de gran interés en su planteamiento y objetivos, tenía un escaso tamaño muestral -tan solo cuatro sujetos-, lo que limitaba enormemente cualquier hallazgo notificado por esta investigación (Turner, 2004).

\subsubsection{RESONANCIA MAGNÉTICA FUNCIONAL}

Los cambios en el flujo cerebral producidos por la percepción musical, medidos a través de resonancia magnética funcional, han sido también una constante en Neurociencia de la Música. A diferencia del electroencefalograma, la resonancia aporta una mayor resolución espacial, por lo que se puede saber con más precisión la región o regiones específicas del cerebro de las que proviene la actividad. Sin embargo, puesto que esta técnica depende del flujo sanguíneo cerebral, existe una pequeña demora en la precisión temporal de los datos, la cual se debe tener en cuenta en los diseños de los estudios de imagen por resonancia magnética.

La mayor parte de las investigaciones que han empleado música de Beethoven y resonancia lo han hecho para apoyar o contradecir con datos de neuroimagen las conclusiones que, el "Efecto Mozart", indicaba en la esfera cognitivo-conductual. Por ejemplo, Bodner y sus colegas refirieron que la Sonata K. 448 de Mozart conducía a una activación de redes importantes para el razonamiento espacial, pero no así la obra "Para Elisa" de Beethoven: entre las diferencias significativas en activación con la composición mozartiana se encontraban la corteza prefrontal dorsolateral, la corteza occipital y el cerebelo (Bodner, Muftuler, Nalcioglu \& Shaw, 2001). 


\section{Miriam AlbusaC Jorge}

Por otro lado y, aunque en este caso no está presente el estímulo puramente auditivo en una parte de la muestra, cabe destacar que cuestiones vinculadas con la "imaginación musical" - es decir, con el hecho de imaginar la música, sin llegar a escucharla- también han implicado a la obra beethoveniana. En un estudio realizado por Zhang y sus colaboradores se elaboró un vídeo sin sonido que, utilizando grafías no convencionales, ofrecía a los participantes pistas visuales sobre el transcurso del primer movimiento de la Sinfonía $n^{\circ}$ 9. Los músicos profesionales debían imaginar la obra, registrándose paralelamente la actividad cerebral. El objetivo era comparar las activaciones cerebrales de personas que escuchaban este primer movimiento frente a las que sólo la imaginaban. Los resultados indicaron que es probable que, en músicos, tanto imaginar la música como la propia percepción de una obra, impliquen activaciones de redes bilaterales (Zhang, Chen, Wen, Lu \& Liu, 2017).

Por último, utilizando la ciencia de las redes se ha querido analizar cómo las preferencias musicales afectan al procesamiento neuronal del arte de los sonidos. Concretamente, en un estudio de Wilkins y sus colegas, se demostró que una importante red cerebral denominada red por defecto -o Default Mode Network en su nombre en lengua inglesa- estaba más conectada a nivel funcional cuando los participantes escuchaban su música preferida (Wilkins, Hodges, Laurienti, Steen \& Burdette, 2014). En esta investigación, los estímulos musicales fueron variados e incluyeron seis piezas, entre las que se encontraba el primer movimiento de la Sinfonía $n^{0} 5$ de Beethoven. Entonces, las conclusiones probaron que, independientemente del género musical escuchado, cuando se trata de una música que el sujeto define como preferida se pueden "experimentar estados emocionales y mentales comparables", aunque el estímulo difiera (Wilkins et al., 2014).

Para concluir este epígrafe y, a modo de resumen, se inserta la siguiente tabla que recopila las obras de Beethoven empleadas como estímulo en la investigación fisiológica y que han sido referidas a lo largo de este artículo.

\begin{tabular}{|c|c|c|c|c|}
\hline Obra & $\begin{array}{c}\text { Duración de } \\
\text { la escucha }\end{array}$ & Registros fisiológicos & No sujetos & $\begin{array}{c}\text { Autores y } \\
\text { año }\end{array}$ \\
\hline $\begin{array}{c}\text { "Para Elisa", } \\
\text { WoO 59 }\end{array}$ & 2 min. (x 3) & $\begin{array}{c}\text { Activación del flujo sanguíneo } \\
\text { cortical medida a través de } \\
\text { resonancia magnética }\end{array}$ & 7 & $\begin{array}{c}\text { Bodner } \text { et al., } \\
2001\end{array}$ \\
\hline $\begin{array}{c}\text { "Para Elisa", } \\
\text { WoO 59 }\end{array}$ & $\begin{array}{c}18 \text { min. -en } \\
\text { bucle- }\end{array}$ & $\begin{array}{c}\text { Actividad eléctrica cerebral medida } \\
\text { a través de electroencefalograma }\end{array}$ & 4 & Turner, 2004 \\
\hline 125. III mov. & $\begin{array}{c}2 \text { min. (x 1) } \\
\text { min. (x 1) }\end{array}$ & $\begin{array}{c}\text { Frecuencia cardíaca, presión } \\
\text { arterial, frecuencia respiratoria, } \\
\text { ventilación, dióxido de carbono, }\end{array}$ & 24 & $\begin{array}{c}\text { Bernardi et } \\
\text { al., 2006 }\end{array}$ \\
\hline
\end{tabular}

${ }^{11} \mathrm{La}$ tabla refleja todos los registros fisiológicos empleados en los estudios, aunque la música no produjera cambios en los mismos. 
LA MÚSICA DE BEETHOVEN EN LA INVESTIGACIÓN FISIOLÓGICA:

DEL ELECTROCARDIOGRAMA A LA IMAGEN POR RESONANCIA MAGNÉTICA

\begin{tabular}{|c|c|c|c|c|}
\hline & & $\begin{array}{l}\text { velocidad del flujo sanguíneo de la } \\
\text { arteria cerebral media y barorreflejo }\end{array}$ & & \\
\hline $\begin{array}{c}\text { Sinfonia } n^{\circ} 2, O p \\
36\end{array}$ & $\begin{array}{l}\text { El tiempo de } \\
\text { realización de } \\
\quad \text { la tarea }\end{array}$ & $\begin{array}{l}\text { Actividad eléctrica cerebral medida } \\
\text { a través de electroencefalograma }\end{array}$ & 16 & $\begin{array}{l}\text { Caldwell \& } \\
\text { Riby, } 2007\end{array}$ \\
\hline $\begin{array}{l}\text { Sonata } n^{\circ} 14, \text { Op. } \\
\qquad 27, n^{\circ} 2 . \\
\text { Romance para violin } \\
n^{\circ} 2, \text { Op. } 50\end{array}$ & Completas & $\begin{array}{c}\text { Frecuencia cardíaca, presión arterial } \\
\text { y temperatura de los dedos }\end{array}$ & 38 & $\begin{array}{l}\text { Lai et al., } \\
2008\end{array}$ \\
\hline $\begin{array}{l}\text { Sinfonia } n^{\circ} \text { 9, Op. } \\
\text { 125. III mov. }\end{array}$ & Mov. completo & $\begin{array}{l}\text { Frecuencia cardíaca, presión } \\
\text { arterial, movimientos respiratorios, } \\
\text { dióxido de carbono, velocidad del } \\
\text { flujo de la arteria cerebral media y } \\
\text { vasomoción cutánea }\end{array}$ & 24 & $\begin{array}{l}\text { Bernardi et } \\
\text { al., } 2009\end{array}$ \\
\hline $\begin{array}{c}\text { Sonata } n^{\circ} 23, O p \\
57\end{array}$ & $\begin{array}{c}\text { Completa } \\
\text { (interpretación) }\end{array}$ & $\begin{array}{l}\text { Frecuencia cardíaca, conductancia } \\
\text { de la piel y actividad muscular }\end{array}$ & 18 & $\begin{array}{c}\text { Yoshie et al., } \\
2009\end{array}$ \\
\hline $\begin{array}{c}\text { Sinfonia } n^{\circ} 5, O p \\
67\end{array}$ & $30 \min .(x)$ & $\begin{array}{c}\text { Frecuencia cardíaca, presión arterial } \\
\text { y frecuencia respiratoria }\end{array}$ & 47 & $\begin{array}{l}\text { Chan et al., } \\
2009\end{array}$ \\
\hline $\begin{array}{l}\text { Sinfonia } n^{0} 5 \text {, Op. } \\
\quad 67 . \mathrm{I} \text { mov. }\end{array}$ & $\begin{array}{l}\text { Mov. completo } \\
\text { (x 2) }\end{array}$ & Actividad eléctrica cerebral & 19 & $\begin{array}{l}\text { Mikutta et al., } \\
2012\end{array}$ \\
\hline $\begin{array}{l}\text { Sinfonia } n^{\circ} 5,0 p \text {. } \\
\quad 67 . \mathrm{I} \text { mov. }\end{array}$ & 5 min. & $\begin{array}{l}\text { Conectividad funcional medida a } \\
\text { través de resonancia magnética }\end{array}$ & 21 & $\begin{array}{l}\text { Wilkins et al., } \\
2014\end{array}$ \\
\hline $\begin{array}{l}\text { Sinfonia } n^{0} 5 \text {, Op. } \\
\text { 67. I mov. }\end{array}$ & $\begin{array}{l}\text { Mov. completo } \\
\quad \text { (x 2) }\end{array}$ & $\begin{array}{l}\text { Actividad eléctrica cerebral medida } \\
\text { a través de electroencefalograma }\end{array}$ & 34 & $\begin{array}{l}\text { Mikutta et al., } \\
2014\end{array}$ \\
\hline $\begin{array}{l}\text { "Para Elisa", } \\
\text { WoO } 59\end{array}$ & Obra completa & $\begin{array}{l}\text { Actividad eléctrica cerebral medida } \\
\text { a través de electroencefalograma }\end{array}$ & 30 & $\begin{array}{l}\text { Verrusio et } \\
\text { al., } 2015\end{array}$ \\
\hline $\begin{array}{l}\text { Sinfonia } n^{\circ} 6, \text { Op. } \\
\quad 68, \text { III mov. } \\
\text { Sonata } n^{\circ} 14, \text { Op. } \\
27, n^{\circ} 2 . \text { I mov. }\end{array}$ & $1 \mathrm{~min}$. & $\begin{array}{l}\text { Actividad eléctrica cerebral medida } \\
\text { a través de electroencefalograma }\end{array}$ & 22 & $\begin{array}{l}\text { Rogenmoser } \\
\text { et al., } 2016\end{array}$ \\
\hline $\begin{array}{l}\text { Sinfonia } n^{\circ} \text { 9, Op. } \\
\text { 125. I mov. }\end{array}$ & 8 min. & $\begin{array}{l}\text { Activación del flujo sanguíneo } \\
\text { cortical medida a través de } \\
\text { resonancia magnética }\end{array}$ & 9 & $\begin{array}{l}\text { Zhang et al., } \\
\qquad 2017\end{array}$ \\
\hline $\begin{array}{c}\text { Sonata } n^{\circ} 14, \text { Op. } \\
27, n^{\circ} 2\end{array}$ & 40 min. & $\begin{array}{c}\text { Frecuencia cardíaca y saturación de } \\
\text { oxígeno }\end{array}$ & 80 & $\begin{array}{l}\text { Rossi et al., } \\
\quad 2018\end{array}$ \\
\hline
\end{tabular}

Tabla 1: Recopilación de las obras de Beethoven que se han empleado en los estudios fisiológicos vinculados con música. 


\section{ConClusiones}

La música de Beethoven se ha empleado en múltiples investigaciones cognitivoconductuales y fisiológicas. En este último caso, sus composiciones han servido como estímulo auditivo en estudios que vinculan la percepción musical con modificaciones de variables que dependen directamente de los sistemas nerviosos central, autónomo y somático. No obstante, dentro de este grueso de publicaciones que utilizan los registros fisiológicos, los estudios que incluyen música de Beethoven no son numerosos y el total de obras de su autoría empleadas no llega a la decena.

En concreto, los géneros incluidos en los estudios son la sonata, la sinfonía, el concierto, el romance y la bagatela, siendo todas las obras instrumentales, tanto para orquesta como para piano solo. Entre estas destaca especialmente la Sinfonía $n^{\circ} 5$, que ha sido la más empleada y recurrente, seguida de la Sinfonía n" 9, la Sonata "Claro de luna" y la Bagatela "Para Elisa", que aparecen con la misma frecuencia. Por tanto, es evidente que las obras que se han utilizado son las más conocidas del compositor y no las del resto de su extenso catálogo, aunque tengan una gran valía musical. Esto puede explicarse por dos razones: en primer lugar, porque los autores de las investigaciones buscan referentes antes de iniciar sus estudios, tomando como base la literatura previa y seleccionando, en muchos casos, obras musicales empleadas con anterioridad que ya han demostrado funcionar para un fin determinado; en segundo término, porque en parte de los estudios analizados era voluntad de los autores seleccionar estímulos que fueran familiares para los participantes.

En lo referente a la duración de los estímulos, hay que poner de relieve que existe una gran variabilidad dependiendo del objetivo de los estudios. Así, algunas obras de Beethoven se han utilizado completas, incluso repitiéndose dos veces o en bucle en caso de que la composición en cuestión no llegara a la duración requerida por la investigación. Sin embargo, otras veces el estímulo musical ha quedado conformado por un único movimiento de la obra en el caso de que esta tuviera varios. Además, en otras ocasiones se han empleado fragmentos muy cortos - de uno o dos minutos, por ejemplo, repitiéndose a veces en varios ensayos-. Así, queda patente que la duración del estímulo no depende directamente de la duración de la obra musical en sí, sino de las necesidades del estudio en cuestión. Por ejemplo, "Para Elisa" se ha empleado repetida en bucle, en una sola audición y en intervalos de escucha de dos minutos repetidos.

En cuanto a la cronología de los trabajos, hay que poner de manifiesto cómo la utilización de la música de Beethoven queda prácticamente reducida a las últimas dos décadas, a pesar de que la trayectoria de los estudios que vinculan la escucha musical con las variables biológicas y su registro tiene más de una centuria. Además, las muestras de participantes utilizadas son, en general, de tamaño medio y especialmente pequeñas en los estudios con resonancia magnética, mientras que los tamaños muestrales más grandes suelen aparecen cuando hay más de dos grupos de sujetos en la investigación.

Resulta significativo también que, en muchas ocasiones, las composiciones beethovenianas se emplean como estímulo control para indagar sobre una hipótesis 
relacionada con la música de Mozart, aunque en la mayor parte de los casos han mostrado efectos similares. Este hecho se debe, en gran medida, a que la música de Mozart ha generado un gran número de publicaciones por la repercusión social que tuvo el llamado "Efecto Mozart", con una serie de adeptos y detractores que han tratado de demostrar las bondades o neutralidad de la música de este compositor. Ante la necesidad de seleccionar un estímulo que sirva como control, probablemente la música de Beethoven se ha elegido porque este último se percibe como otro de los grandes compositores de la historia de la música, además de ser coetáneo a Mozart.

En lo que concierne a las técnicas de registro fisiológico empleadas, cabe señalar que destacan las que utilizan música de Beethoven para rastrear las actividades cardiovascular y respiratoria, junto con el electroencefalograma. Este último se ha preferido a la resonancia magnética como técnica para el análisis de la actividad cerebral, probablemente por el elevado coste que suponen los estudios de resonancia.

Los hallazgos de los estudios muestran que la música de Beethoven, junto con la de otros autores, tiene un efecto e influencia en las respuestas fisiológicas, lo cual podría tener implicaciones en el ámbito clínico. La modulación de variables como la frecuencia cardíaca, el pulso o la frecuencia respiratoria, ofrecen la posibilidad de incorporar la música de Beethoven y de otros compositores en una amplia gama de estrategias de intervención, por ejemplo, para reducir el estrés. No obstante, sería necesario hacer más investigaciones en este sentido.

Por último, es importante señalar que, aunque las composiciones de Beethoven han mostrado producir efectos sobre el organismo, estos han aparecido también ante la escucha de otras músicas, lo que podría inducir a pensar que son los atributos musicales tales como el tempo, el ritmo, la intensidad, la estructura o la tonalidad, junto con otros factores de relevancia como la preferencia musical, los que determinan el tipo de influencia en el ser humano, más que el compositor de la música en sí. De hecho, en diversos estudios en los que las composiciones empleadas tenían características musicales compartidas, los efectos producidos sobre la fisiología eran similares, independientemente del género o la autoría. Además, las personas que escuchan su música preferida pueden experimentar emociones y estados mentales similares, aún cuando los estímulos de partida son distintos. En conclusión, los registros fisiológicos indican la influencia de la música de Beethoven en las funciones humanas, siendo esta dependiente, entre otras cuestiones, de las características y cualidades musicales concretas de cada obra, las emociones vinculadas y/o producidas por ella y las preferencias individuales de los sujetos. 


\section{Miriam AlbuSAC Jorge}

\section{BIBLIOGRAFÍA}

Abecasis, D., Brochard, R., Del Río, D., Dufour, A. \& Ortiz, T. (2009). Brain lateralization of metrical accenting in musicians. Annals of the New York. Academy of Sciences, 1169(1), pp. 74-78. Doi: 10.1111/j.1749-6632.2009.04766.x. Recuperado de https://tinyurl.com/y6smrvly

Aoun, P., Jones, T., Shaw, G. L. \& Bodner, M. (2005). Long-term enhancement of maze learning in mice via a generalized Mozart effect. Neurological Research, 27(8), pp. 791796. Doi: 10.1179/016164105X63647. Recuperado de https://tinyurl.com/y4q536bh

Barquero Jiménez, M. S. \& Payno Vargas, M.A. (2001). Las amusias. Revista de Neurología, 32(5), pp. 454-462. Doi: 10.33588/rn.3205.2000168. Recuperado de https://tinyurl.com/y6spgg94

Beethoven, L. V. (1933). Epistolario de Beethoven [Barrado, A. (traductor)]. Madrid: Poblet.

Berlioz, H. (1844). Voyage musical en Allemagne et en Italie. Etudes sur Beethoven, Glück et Weber. París: Jules Labitte.

Bernardi, L., Porta, C. \& Sleight, P. (2006). Cardiovascular, cerebrovascular, and respiratory changes induced by different types of music in musicians and non-musicians: the importance of silence. Heart, 92(4), pp. 445-452. Doi: 10.1136/hrt.2005.064600. Recuperado de https://tinyurl.com/y4uykvwz

Bernardi, L., Porta, C., Casucci, G., Balsamo, R., Bernardi, N. F., Fogari, R. \& Sleight, P. (2009). Dynamic interactions between musical, cardiovascular, and cerebral rhythms in humans. Circulation, 119(25), pp. 3171-3180. Doi: 10.1161/CIRCULATIONAHA.108.806174.

Recuperado de https://tinyurl.com/yyne $5 \mathrm{k} 63$

Bodner, M., Muftuler, L. T., Nalcioglu, O. \& Shaw, G. L. (2001). fMRI study relevant to the mozart effect: Brain areas involved in spatial-temporal reasoning. Neurological Research, 23(7), pp. 683-690. Doi: 10.1179/016164101101199108. Recuperado de https://tinyurl.com/y3voxmk4

Bullack, A., Büdenbender, N., Roden, I. \& Kreutz, G. (2018). Psychophysiological Responses to "Happy" and "Sad" Music: A Replication Study. Music Perception, 35(4), pp. 502-517. Doi: 10.1525/mp.2018.35.4.502. Recuperado de https://tinyurl.com/yyry32gm 
LA MÚSICA DE BEETHOVEN EN LA INVESTIGACIÓN FISIOLÓGICA:

DEL ELECTROCARDIOGRAMA A LA IMAGEN POR RESONANCIA MAGNÉTICA

Caldwell, G. N. \& Riby, L. M. (2007). The effects of music exposure and own genre preference on conscious and unconscious cognitive processes: A pilot ERP study. Consciousness and Cognition, 16(4), pp. 992-996. Doi: 10.1016/j.concog.2006.06.015. Recuperado de https://tinyurl.com/y6re5uuo

Carmona, E. \& Daza, M. T. (2008). La percepción musical. En D. Alonso Cánovas, A. F. Estévez y F. Sánchez-Santed (Eds.). El cerebro musical (pp. 73-96). Almería: Universidad de Almería.

Chan, M. F., Chan, E. A., Mok, E. \& Kwan Tse, F. Y. (2009). Effect of music on depression levels and physiological responses in community-based older adults. International Journal of Mental Health Nursing, 18(4), pp. 285-294. Doi: 10.1111/j.1447-0349.2009.00614.x. Recuperado de https://tinyurl.com/yxrcupax

Chen, J. L., Penhume, V. B. \& Zatorre, R. J. (2009). The role of auditory and premotor cortex in sensorimotor transformations. Annals of the New York. Academy of Sciences, 1169(1), pp. 15-34. Doi: 10.1111/j.1749-6632.2009.04556.x. Recuperado de https://tinyurl.com/yxtgprqd

Chan, P. Y., Dong, M. \& Li, H. (2019). The Science of Harmony: A Psychophysical Basis for Perceptual Tensions and Resolutions in Music. Research, 2369041. Doi: 10.34133/2019/2369041. Recuperado de https://tinyurl.com/y5lzt9ly

Clynes, M. (1995). Microstructural musical linguistics: composers' pulses are liked most by the best musicians. Cognition, 55(3), pp. 269-310. Doi: 10.1016/0010-0277(94)00650A. Recuperado de https://tinyurl.com/y2mcpsvg

Cooper, A. Z., Nair, S. S. \& Tremaglio, J. M. (2016). Diagnosing Beethoven: A New Take on an Old Patient. The American journal of medicine, 129(1), pp. 126-127. Doi: 10.1016/j.amjmed.2015.07.030. Recuperado de https://tinyurl.com/yx8rkt3h

Daikoku T. (2018). Time-course variation of statistics embedded in music: Corpus study on implicit learning and knowledge. Plos One, 13(5), e0196493. Doi: 10.1371/journal.pone.0196493. Recuperado de https://tinyurl.com/y3zf46ec

Daikoku, T. (2019a). Depth and the Uncertainty of Statistical Knowledge on Musical Creativity Fluctuate Over a Composer's Lifetime. Frontiers in Computational Neuroscience, 13. Doi: 10.3389/fncom.2019.00027. Recuperado de https://tinyurl.com/y4qnpj6y 


\section{Miriam AlbusaC Jorge}

Daikoku, T. (2019b). Statistical learning and the uncertainty of melody and bass line in music. Plos one, 14(12), e0226734. Doi: 10.1371/journal.pone.0226734. Recuperado de https://tinyurl.com/y4euquck

Dahlhaus, C. (1987). Ludwig van Beethoven und seine Zeit. Laaber: Laaber-Verlag.

DeNora, T. (1995.) Beethoven and the Construction of Genius: Musical Politics in Vienna, 1792-1803. Berkeley: University of California Press.

Dosseville, F., Laborde, S. \& Scelles, N. (2012). Music during lectures: Will students learn better?. Learning and Individual Differences, 22(2), pp. 258-262. Doi: 10.1016/j.lindif.2011.10.004. Recuperado de https://tinyurl.com/y5ynbdjw

Davey, B. (productor) \& Rose, B. (director) (1994). Immortal beloved. Estados Unidos: Columbia Pictures.

Eitan, Z. \& Timmers, R. (2010). Beethoven's last piano sonata and those who follow crocodiles: Cross-domain mappings of auditory pitch in a musical context. Cognition, 114(3), pp. 405-422. Doi: 10.1016/j.cognition.2009.10.013. Recuperado de https://tinyurl.com/y4smdam5

Ellis, D. \& Brighouse, G. (1952). Effects of Music on Respiration- and Heart-Rate. The American Journal of Psychology, 65(1), pp. 39-47. Doi:10.2307/1418826. Recuperado de https://tinyurl.com/yxf2o4bg

Enghels, R. \& Roegiest, E. (2004). Percepción visual y percepción auditiva: la naturaleza del objeto. En E. Serra \& G. Wotjak (Eds.), Cognición y percepción lingü̈sticas (pp. 47- 59). Valencia: Guada.

Gao, C., Fillmore, P. \& Scullin, M. K. (2020). Classical music, educational learning, and slow wave sleep: A targeted memory reactivation experiment. Neurobiology of Learning and Memory, 171, 107206. Doi: 10.1016/j.nlm.2020.107206. Recuperado de https://tinyurl.com/yxe3f47t

Gasenzer, E. R. \& Neugebauer, E. A. (2015). Genie zwischen Kunst und Krankheit: Medizinische Betrachtungen zu Ludwig van Beethoven [Genius between music and disease: Medical considerations on Ludwig van Beethoven]. Wiener medizinische Wochenschrift, 165(21-22), pp. 445-457. Doi: 10.1007/s10354-015-0393-2. Recuperado de https://tinyurl.com/yyf8q9sh 
LA MÚSICA DE BEETHOVEN EN LA INVESTIGACIÓN FISIOLÓGICA:

DEL ELECTROCARDIOGRAMA A LA IMAGEN POR RESONANCIA MAGNÉTICA

Giannouli, V., Tsolaki, M. \& Kargopoulos, P. (2010). The influence of Mozart's and Beethoven's music on reverse mnemonic recall tasks. Psychiatriki, 21(1), pp. 60-67.

Grahn, J. A. (2009). The role of the basal ganglia in beat perception: neuroimaging and neuropsychological investigations. Annals of the New York. Academy of Sciences, 1169(1), pp. 35-45. Doi: 10.1111/j.1749-6632.2009.04553.x. Recuperado de https://tinyurl.com/y2ugwtgb

Grahn, J. A. (2012). Neural mechanisms of rhythm perception: current findings and future perspectives. Topics in cognitive science, 4(4), pp. 585-606. Doi: 10.1111/j.17568765.2012.01213.x. Recuperado de https://tinyurl.com/y3abh7ss

Grewe, O., Nagel, F., Kopiez, R. \& Altenmüller, E. (2007). Emotions over time: synchronicity and development of subjective, physiological, and facial affective reactions to music. Emotion, 7(4), pp. 774-788. Doi: 10.1037/1528-3542.7.4.774. Recuperado de https://tinyurl.com/y6bhvxhm

Hamada, M., Zaidan, B. B. \& Zaidan, A. A. (2018). A Systematic Review for Human EEG Brain Signals Based Emotion Classification, Feature Extraction, Brain Condition, Group Comparison. Journal of Medical Systems volume, 42, 162. Doi: 10.1007/s10916018-1020-8. Recuperado de https://tinyurl.com/y4n6qmg7

Hausmann, M., Hodgetts, S. \& Eerola, T. (2016). Music-induced changes in functional cerebral asymmetries. Brain and Cognition, 104, pp. 58-71. Doi: 10.1016/j.bandc.2016.03.001. Recuperado de https://tinyurl.com/y3wy8vwt

Herremans, D. \& Chuan, C. (2017). A Multi-modal Platform for Semantic Music Analysis: Visualizing Audio-and Score-Based Tension. 2017 IEEE 11th International Conference on Semantic Computing (ICSC) (pp. 419-426). San Diego: IEEE Computer Society. Doi: 10.1109/ICSC.2017.49. Recuperado de https://tinyurl.com/yxh7jy2q

Hodges, D. A. (2010). Psychophysiological measures. En P. N. Juslin \& J. A. Sloboda (Eds.), Series in affective science. Handbook of music and emotion: Theory, research, applications (pp. 279-311). Oxford: Oxford University Press.

Hurless, N., Mekic, A., Peña, S., Humphries, E., Gentry, H. \& Nichols, D. F. (2013). Impulse: The Premier Undergraduate Neuroscience Journal. Recuperado de https://tinyurl.com/yy56evfx 


\section{Miriam AlbusaC Jorge}

Huxtable, R. J. (2001). Beethoven: a life of sound and silence. Molecular interventions, 1(1), pp. 8-12.

Hyde, I. H. (1924). Effects of Music Upon Electrocardiograms and Blood Pressure. Journal of Experimental Psychology, 7(3), pp. 213-224. Doi: 10.1037/h0073580.

Hyde, I. H. \& Scalapino, W. (1918). The influence of music upon electrocardiograms and blood pressure. American Journal of Physiology, 46(1), pp. 35-38. Doi: 10.1152/ajplegacy.1918.46.1.35. Recuperado de https://tinyurl.com/y46mqwgu

Iwanaga, M. \& Moroki, Y. (1999). Subjective and Physiological Responses to Music Stimuli Controlled Over Activity and Preference. Journal of Music Therapy, 36(1), pp. 26-38. Doi: 10.1093/jmt/36.1.26. Recuperado de https://tinyurl.com/yxwpkmy4

Khalfa, S., Peretz, I., Blondin J. P. \& Robert, M. (2002). Event-related skin conductance responses to musical emotions in humans. Neuroscience Letters, 328(2), pp. 145-149. Doi: 10.1016/S0304-3940(02)00462-7. Recuperado de https://tinyurl.com/y4am3v4u

Karmody, C. S. \& Bachor, E. S. (2005). The Deafness of Ludwig Van Beethoven: an Immunopathy. Otology \& Neurotology, 26(4), pp. 809-814. Doi: 10.1097/01.mao.0000178149.36881.df. Recuperado de https://tinyurl.com/y28t45g8

Kiernan, J. A. (2013). Barr. El sistema nervioso humano: una perspectiva anatómica (9a. ed.). Barcelona: Wolters Kluwer Health.

Kivy, P. (2001). The Possessor and the Possessed: Handel, Mozart, Beethoven and the Idea of Musical Genius. New Haven: Yale University Press.

Koelsch S. (2011). Toward a neural basis of music perception - a review and updated model. Frontiers in psychology, 2, 110. Doi: 10.3389/fpsyg.2011.00110. Recuperado de https://tinyurl.com/y66akubl

Koelsch, S. \& Siebel, W. A. (2005). Towards a neural basis of music perception. Trends in Cognitive Sciences, 9(12), pp. 578-584. Doi: 10.1016/j.tics.2005.10.001. Recuperado de https://tinyurl.com/y2c43nuu

Kozbelt A. (2007). A quantitative analysis of Beethoven as self-critic: implications for psychological theories of musical creativity. Psychology of Music, 35(1), pp. 144-168. Doi: 10.1177/0305735607068892. Recuperado de https://tinyurl.com/y63gvmh9 
LA MÚSICA DE BEETHOVEN EN LA INVESTIGACIÓN FISIOLÓGICA:

DEL ELECTROCARDIOGRAMA A LA IMAGEN POR RESONANCIA MAGNÉTICA

Krumhansl, C. L. (1997). An Exploratory Study of Musical Emotions and Psychophysiology. Canadian Journal of Experimental Psychology, 51(4), pp. 336-352. Doi: 10.1037/11961961.51.4.336. Recuperado de https://tinyurl.com/y5ah9s6t

Lai, H.-L., Chen, P-W., Chen, C.-J., Chang, H.-K., Peng, T.-C. \& Chang, F.-M. (2008). Randomized crossover trial studying the effect of music on examination anxiety. Nurse Education Today, 28(8), pp. 909-916. Doi: 10.1016/j.nedt.2008.05.011. Recuperado de https://tinyurl.com/yyuwsne8

Lalitte, P., Bigand, E., Kantor-Martynuska, J. \& Delbé, C. (2009). On Listening to Atonal Variants of Two Piano Sonatas by Beethoven. Music Perception: An Interdisciplinary Journal, 26(3), pp. 223-234. Doi: 10.1525/mp.2009.26.3.223. Recuperado de https://tinyurl.com/yxwhrhlm

Levitin, D. (2008). Tu cerebro y la música: el estudio científico de una obsesión bumana. Barcelona: RBA.

Lundqvist, L-O, Carlsson, F., Hilmersson, P. \& Juslin, P. N. (2009). Emotional responses to music: experience, expression, and physiology. Psychology of Music, 37(1), pp. 61-90. Doi: 10.1177/0305735607086048. Recuperado de https://tinyurl.com/y3wmapzq

Marshall, L. (productor) \& Cellan Jones, S. (director) (2003). Eroica. Londres: BBC.

Martín Moreno, A. (2003). Música, pasión, razón: la teoría de los afectos en el teatro y la música del siglo de Oro. Edad de Oro, 22, pp. 321-360.

Merrill, J., Omigie, D. \& Wald-Fuhrmann, M. (2020). Locus of emotion influences psychophysiological reactions to music. Plos One, 15(8), e0237641. Doi: 10.1371/journal.pone.0237641. Recuperado de https://tinyurl.com/y59v9h9o

Mikutta, C., Altorfer, A., Strik, W. \& Koenig, T. (2012). Emotions, arousal, and frontal alpha rhythm asymmetry during beethoven's 5th symphony. Brain Topography, 25(4), pp. 423430. Doi: 10.1007/s10548-012-0227-0. Recuperado de https://tinyurl.com/y3qchtrm

Mikutta, C. A., Maissen, G., Altorfer, A., Strik, W. \& Koenig, T. (2014). Professional musicians listen differently to music. Neuroscience, 268, pp. 102-111. Doi: 10.1016/j.neuroscience.2014.03.007. Recuperado de https://tinyurl.com/y5v8n47q 


\section{Miriam AlbusaC Jorge}

Mitas, A. W., Lipowicz, A. M., Wijata, A. M. \& Bieńkowska, M. J. (2016). Impact of Music on the Effectiveness of Performing Mathematical-Logical Tasks. En E. Piętka, P. Badura, J. Kawa \& W. Wieclawek (Eds.), Information Technologies in Medicine. Advances in Intelligent Systems and Computing, 471 (pp. 409-420). Springer. Doi: 10.1007/978-3-31939796-2_34. Recuperado de https://tinyurl.com/y6gal757

Montes-Santiago, J. (2013). The lead-poisoned genius: saturnism in famous artists across five centuries. Progress in brain research, 203, pp. 223-240. Doi: 10.1016/B978-0-444-627308.00009-8. Recuperado de https://tinyurl.com/y5knaxht

Montilla López, P. (1999). El cerebro y la música: un enfoque interdisciplinario. Córdoba: Servicio de publicaciones de la Universidad de Córdoba.

Moss, F. C., Neuwirth, M., Harasim, D. \& Rohrmeier, M. (2019). Statistical characteristics of tonal harmony: A corpus study of Beethoven's string quartets. Plos One 14(6), e0217242. Doi: 10.1371/journal.pone.0217242. Recuperado de https://tinyurl.com/y2psefc8

Nyklíček, I., Thayer, J. F. \& Van Doornen, L. J. P. (1997). Cardiorespiratory Differentiation of Musically-Induced Emotions. Journal of Psychophysiology, 11(4), pp. 304-321. Recuperado de https://tinyurl.com/y35fpobl

Orini, M., Bailón, R., Enk, R., Koelsch, S., Mainardi, L. \& Laguna, P. (2010). A method for continuously assessing the autonomic response to music-induced emotions through HRV analysis. Medical \& biological engineering \& computing, 48(5), pp. 423-433. Doi: 10.1007/s11517-010-0592-3. Recuperado de https://tinyurl.com/y2ef3gmw

Palau, J. (1945). La vida de Beethoven. Barcelona: Seix Barral.

Patterson, R. D., Uppenkamp, S., Johnsrude, I. S. \& Griffiths, T. D. (2002). The processing of temporal pitch and melody information in auditory cortex. Neuron, 36(4), pp. 767776. Doi: 10.1016/s0896-6273(02)01060-7. Recuperado de https://tinyurl.com/y3oo28du

Perciaccante, A., Coralli, A. \& Bauman, N. G. (2020). Beethoven: His Hearing Loss and His Hearing Aids. Otology \& neurotology, 41(9), pp. 1305-1308. Doi: 10.1097/MAO.0000000000002755. Recuperado de https://tinyurl.com/y67xmres

Peretz, I. \& Coltheart, M. (2003). Modularity of music processing. Nature neuroscience, 6(7), pp. 688-691. Doi: 10.1038/nn1083. Recuperado de https://tinyurl.com/y4buovx4 
LA MÚSICA DE BEETHOVEN EN LA INVESTIGACIÓN FISIOLÓGICA:

DEL ELECTROCARDIOGRAMA A LA IMAGEN POR RESONANCIA MAGNÉTICA

Peretz, I., Vuvan, D., Lagrois, M. É. \& Armony, J. L. (2015). Neural overlap in processing music and speech. Philosophical transactions of the Royal Society of London. Series B, Biological sciences, 370(1664), 20140090. Doi: 10.1098/rstb.2014.0090. Recuperado de https://tinyurl.com/y6b6kvvv

Peretz, I. \& Zatorre, R. J. (2005). Brain organization for music processing. Annual review of psychology, 56, pp. 89-114. Doi: 10.1146/annurev.psych.56.091103.070225. Recuperado de https://tinyurl.com/yxwa7m4t

Poggi, A. \& Vallora, E. (1995). Beethoven: signori, il catalogo è questo!. Torino: Einaudi.

Rauscher, F. H., Shaw, G. L. \& Ky, C. N. (1993). Music and spatial task performance. Nature, 365(6447), pp. 611-611. Doi: 10.1038/365611a0. Recuperado de https://tinyurl.com/y6zdftk3

Real Academia Española (2019). Diccionario de la lengua española, 23. ${ }^{a}$ ed. Recuperado de https://dle.rae.es

Redolar Ripoll, D. (Coord.) (2010). Fundamentos de psicobiología. Barcelona: Universitat Oberta de Catalunya.

Repp, B. H. (1990). Patterns of expressive timing in performances of a Beethoven minuet by nineteen pianists. The Journal of the Acoustical Society of America, 88(2), pp. 622-641. Doi: 10.1121/1.399766. Recuperado de https://tinyurl.com/y5j4ucek

Rogenmoser, L., Zollinger, N., Elmer, S. \& Jäncke, L. (2016). Independent component processes underlying emotions during natural music listening. Social Cognitive and Affective Neuroscience, 11(9), pp. 1428-1439. Doi: 10.1093/scan/nsw048. Recuperado de https://tinyurl.com/yxp5538q

Rolland, R. (1915). Vida de Beethoven. Madrid: Publicaciones de la Residencia de Estudiantes.

Rossi, A., Molinaro, A., Savi, E., Micheletti, S., Galli, J., Chirico \& Fazzi, E. (2018). Music reduces pain perception in healthy newborns: A comparison between different music tracks and recoded heartbeat. Early Human Development, 124, pp. 7-10. Doi: 10.1016/j.earlhumdev.2018.07.006. Recuperado de https://tinyurl.com/y3j94rmk

Samson, S. (2003). Neuropsychological Studies of Musical Timbre. Annals of the New York Academy of Sciences, 999(1), pp. 144-151. Doi: 10.1196/annals.1284.016. Recuperado de https://tinyurl.com/y3wf5ggh 


\section{Miriam Albusac Jorge}

Servant, I. \& Baccino, T. (1999). Lire Beethoven: une étude exploratoire des mouvements des yeux [Reading Beethoven: An exploratory study of eye movement]. Musica Scientice, 3(1), pp. 67-94. Doi: 10.1177/102986499900300104. Recuperado de https://tinyurl.com/y2f5z291

Smith, N. A. \& Cuddy, L. L. (2003). Perceptions of musical dimensions in Beethoven's Waldstein sonata: An application of Tonal Pitch Space theory. Musicae Scientiae, 7(1), pp. 7-31. Doi: 10.1177/102986490300700102. Recuperado de https://tinyurl.com/y6x4tw2v

Snyder, J. S., Large, E. W. \& Penhune, V. (2009). Part I Introduction: Rhythms in the brain: basic science and clinical perspectives. Annals of the New York. Academy of Sciences, 1169(1), pp. 13-14. Doi: 10.1111/j.1749-6632.2009.04860.x. Recuperado de https://tinyurl.com/y67f2ahf

Son, H. K., So, W. Y. \& Kim, M. (2019). Effects of Aromatherapy Combined with Music Therapy on Anxiety, Stress, and Fundamental Nursing Skills in Nursing Students: A Randomized Controlled Trial. International journal of environmental research and public bealth, 16(21), 4185. Doi: 10.3390/ijerph16214185. Recuperado de https://tinyurl.com/y47w3shd

Suda, M., Morimoto, K., Obata, A., Koizumi, H. \& Maki, A. (2008). Cortical responses to Mozart's sonata enhance spatial-reasoning ability. Neurological Research, 30(9), pp. 885888. Doi: 10.1179/174313208X319143. Recuperado de https://tinyurl.com/yxgxslx7

Tillmann, B., Lalitte, P., Albouy, P., Caclin, A. \& Bigand, E. (2016). Discrimination of tonal and atonal music in congenital amusia: The advantage of implicit tasks. Neuropsychologia, 85, pp. 10-18. Doi: 10.1016/j.neuropsychologia.2016.02.027. Recuperado de https://tinyurl.com/yybutt6u

Timmers, R., Ashley, R., Desain, P., Honing, H. \& Windsor, W. L. (2002). Timing of Ornaments in the Theme from Beethoven's Paisiello Variations: Empirical Data and a Model. Music Perception, 20(1), pp. 3-33. Doi: 10.1525/mp.2002.20.1.3. Recuperado de https://tinyurl.com/y2tdnzjx

Trappe, H.-J. (2010). The effects of music on the cardiovascular system and cardiovascular health. Heart, 96(23), pp. 1868-1871. Doi: 10.1136/hrt.2010.209858. Recuperado de https://tinyurl.com/y3bmn4x9 
LA MÚSICA DE BEETHOVEN EN LA INVESTIGACIÓN FISIOLÓGICA:

DEL ELECTROCARDIOGRAMA A LA IMAGEN POR RESONANCIA MAGNÉTICA

Trappe, H.-J. (2012a). Music and medicine: The effects of music on the human being. Applied Cardiopulmonary Pathophysiology, 16(2), pp. 133-142. Doi: 10.1179/174313208X319143. Recuperado de https://tinyurl.com/cgoex83

Trappe H.-J. (2012b). Role of music in intensive care medicine. International journal of critical illness and injury science, 2(1), pp. 27-31. Doi: 10.4103/2229-5151.94893. Recuperado de https://tinyurl.com/y3btltml

Turner, R. P. (2004). The acute effect of music on interictal epileptiform discharges. Epilepsy and Behavior, 5(5), pp. 662-668. Doi: 10.1016/j.yebeh.2004.07.003. Recuperado de https://tinyurl.com/yxgy6j2s

Verrusio, W., Ettorre, E., Vicenzini, E., Vanacore, N., Cacciafesta, M. \& Mecarelli, O. (2015). The Mozart Effect: A quantitative EEG study. Consciousness and Cognition, 35, pp. 150155. Doi: 10.1016/j.concog.2015.05.005. Recuperado de https://tinyurl.com/y33pdwyr

Wagner, R. (1870). Beethoven. Leipzig: Insel-Verlag.

Warren, J. D. (2008). How does the brain process music?. Clinical medicine, 8(1), pp. 32-36. Doi: 10.7861/clinmedicine.8-1-32. Recuperados de https://tinyurl.com/yyauon79

Wilkins, R. W., Hodges, D. A., Laurienti, P. J., Steen, M. \& Burdette, J. H. (2014). Network science and the effects of music preference on functional Brain connectivity: From Beethoven to Eminem. Scientific Reports, 4, 6130. Doi: 10.1038/srep06130. Recuperado de https://tinyurl.com/kzfxpkd

Wilkinson, C. (productor) \& Holland, A. (director) (2006). Copying Beethoven. United States: Metro-Goldwyn-Mayer.

Yoshie, M., Kudo, K., Murakoshi, T. \& Ohtsuki. (2009). Music performance anxiety in skilled pianists: effects of social-evaluative performance situation on subjective, autonomic, and electromyographic reactions. Experimental Brain Research volume, 199, 117. Doi: 10.1007/s00221-009-1979-y. Recuperado de https://tinyurl.com/y29vbkfg

Zatorre, R. J. (1988). Pitch perception of complex tones and human temporal lobe function. Journal of the acoustical society of America, 84(2), pp. 566-572. Doi: 10.1121/1.396834. Recuperado de https://tinyurl.com/y58c8f2m 


\section{Miriam Albusac Jorge}

Zatorre, R. J. (2001). Neural specializations for tonal processing. Annals of the New York Academy of Sciences, 930(1), pp. 193-210. Doi: 10.1111/j.1749-6632.2001.tb05734.x. Recuperado de https://tinyurl.com/y6d4vmfd

Zhang, Y., Chen, G., Wen, H., Lu, K-H. \& Liu, Z. (2017). Musical Imagery Involves Wernicke's Area in Bilateral and Anti-Correlated Network Interactions in Musicians. Scientific Reports, 7, 17066. Doi: 10.1038/s41598-017-17178-4. Recuperado de https://tinyurl.com/yyalxohv

Fecha de recepción: 02/11/2020

Fecha de aceptación: 17/11/2020 